\title{
Multi-channel, microwave-based, compact printed sensor for simultaneous and independent level measurement of eight liquids
}

\author{
Muhammad Akram Karimi, Student Member, IEEE, Muhammad Arsalan, and Atif Shamim, Senior Member, IEEE
}

\begin{abstract}
Industrial automation relies on sensing the process parameters like liquid levels. Simultaneous sensing of multiple fluids is especially useful for many automation processes involving multiple ingredients. Some of the desirable features in any level sensor are its non-intrusiveness, high accuracy and consistent performance. These features can be achieved with typical microwave radar based level sensors. However, this paper presents a microwave resonance method instead of typical microwave reflectance (radar) to reliably sense the liquid level at fraction of the cost using a unique printed sensor. In addition to that, sensor design presented in this article enables sensing liquid level of 8 liquids simultaneously and independently utilizing its multiple channels. An array of eight modified microwave $T$ resonators has been compactly packed inside a metallic cylinder of just 4" diameter where the resonators share the same ground plane. Sensors' performance, to measure the unknown level of eight different fluids (with dielectric constant $\left(\epsilon_{\mathrm{r}}\right)$ ranging from 2.8 to 80), has been measured in real time using a custom-built software. Simple one point calibration procedure has also been proposed in this paper which has resulted into measurement accuracy of $\sim 0.6 \%$ which is better than $1 \%$, realizable with highend and expensive radar based level sensors.
\end{abstract}

Index Terms-3D Printing, Level Sensor, Microwave TResonator, Screen printing

\section{INTRODUCTION}

A UTOMATION has increased the industrial efficiency manifolds. Process engineering has become crucial for optimizing production processes, cutting costs, saving energy and minimizing the ecological risks. Industries have come a long way to achieve all this with various industrial sensors acting as a backbone of the process.

Wide range of industrial applications require to store different liquids in storage tanks made of metal. It's crucial to sense the real time liquid (or granules) level for industrial process automation. Wide range of sensors based upon the principles of optics [1], ultrasounds [2] [3], capacitance [4] [5] and radars [6] [7] are currently available to do the job. However, optical sensors suffer with low signal-to-noise (SNR) ratio in the presence of dust or fog while ultrasound based level sensors are known to get affected by the surface foams [8]. It has been shown by Demori in [9] that low excitation frequency (below 2

Manuscript received December 12, 2018; revised Jan 22, 2019 (Corresponding author: Muhammad Akram Karimi).

M. A. Karimi and A. Shamim are with Integrated Microwave Packaging Antennas and Circuits Technology, Computer, Electrical, and Mathematical Sciences and Engineering Division, King Abdullah
$\mathrm{MHz}$ ), which is typically used to extract capacitance to sense liquids based upon their dielectric constant, results into low sensitivity especially in the presence of lossy mediums like saline water. High excitation frequency is therefore recommended while sensing fluids based upon their dielectric constant. Traditionally, microwave resonator based sensors are used for characterizing small amounts of fluids in microfluidic channels [10][11]. For high-volume liquid sensing, ultra-high frequency guided wave radar based level sensors are currently used which are not only expensive [12] but are intrusive as well [7] while contactless radar based level sensors are affected by surface turbulence during liquid agitation and extensive signal processing is required to mitigate the effects which increases the cost of the system [8].

Many industries including food [13] and plastic chemicals [14] require precise mixing of different ingredients including liquids, gels or granules as per the optimum recipes. Currently, separate sensor is installed on each and every ingredient increasing the footprint of the automated feeding system. However, this article presents a sensor capable of sensing the level of eight different materials (ingredients) simultaneously and independently in a very compact fashion. This will enable to reduce the size of the automation system manifolds.

In addition to simultaneous sensing of multiple fluids, our proposed multi-sensor makes use of radio frequency, in the range of $130 \mathrm{MHz}$ to $180 \mathrm{MHz}$, which is much higher in frequency as compared to the excitation frequency used for capacitive sensors, while it is much lower in frequency than what is used for radar based level sensors making its readout circuitry much cheaper and easier to realize. In radar based wave reflective sensors, resolution is directly linked with the frequency used because of which $24 \mathrm{GHz}$ frequency band is typically used to achieve $1 \%$ resolution [8]. In comparison, the presented resonator based microwave sensor has been demonstrated to achieve level measurement accuracy of $0.6 \%$ and the resolution at least 5 times higher i.e. around $0.1 \%$.

Moreover, the proposed level measurement system requires simple time-based averaging to mitigate the effects of turbulence caused due to agitation. Some unshielded nonmetallic microwave level and dielectric properties sensors can also be found in literature [15] [16] [17] [18] [19] which in contrast to our design can't be used in metallic enclosures which are typically found in industry.

\footnotetext{
University of Science and Technology, Thuwal 23955, Saudi Arabia (email: muhammadakram.karimi@kaust.edu.sa).

M. Arsalan is with the Production Technology Team, EXPEC ARC, Saudi Aramco 31311, Saudi Arabia (e-mail: muhammad.arsalan@aramco.com)
} 


\section{SENSING PRINCIPLE}

The sensor presented in this article exploit the fact that the microwaves slow down while passing through any dielectric medium other than air by a factor of $\sqrt{\varepsilon_{r} \mu_{r}}$ where $\varepsilon \mathrm{r}$ and $\mu \mathrm{r}$ are the relative permittivity and relative permeability of the medium [20].

The proposed sensor is fundamentally based upon the principle of microwave T-resonance. Typical T-resonator consists of a series feedline (FL) to feed and analyze the microwave signals which is connected to a $\lambda / 4$ shunt stub (SS) acting as resonator. FL and SS lies on one side of the flat substrate with a common ground plane on the other side of it [21]. $\quad \lambda / 4$ shunt stub can simply be thought of distributed inductance (L) and capacitance (C) as shown in Fig. 1. As we know that LC resonant tank resonates at a resonant frequency " $\mathrm{f}_{0}$ " which is inversely proportional to $\sqrt{\mathrm{C}}$ as given by eq. 1 . Moreover, the capacitance is directly proportional to the effective dielectric constant ( $\varepsilon$ eff) as given by eq. 2 . Combining eq. (1) and (2) with the simplified model of the T-resonator, we can say that as the resonator's resonance frequency should decrease with increasing dielectric constant of the medium present between shunt stub and its ground plane.

$$
\begin{gathered}
\text { fo }=1 /(2 \Pi \sqrt{\mathrm{LC}}) \\
C \propto \in_{\text {eff }}
\end{gathered}
$$

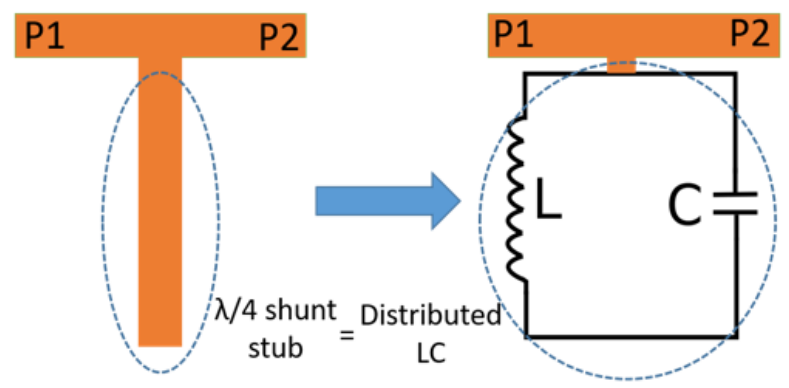

Fig. 1. Simplified equivalent circuit of T resonator, where P1 and P2 represent input and output ports respectively

Air has the lowest relative permittivity i.e. $(\varepsilon r=1)$ in contrast to any other dielectric medium ( $\varepsilon r>1)$; so the T-resonator facing
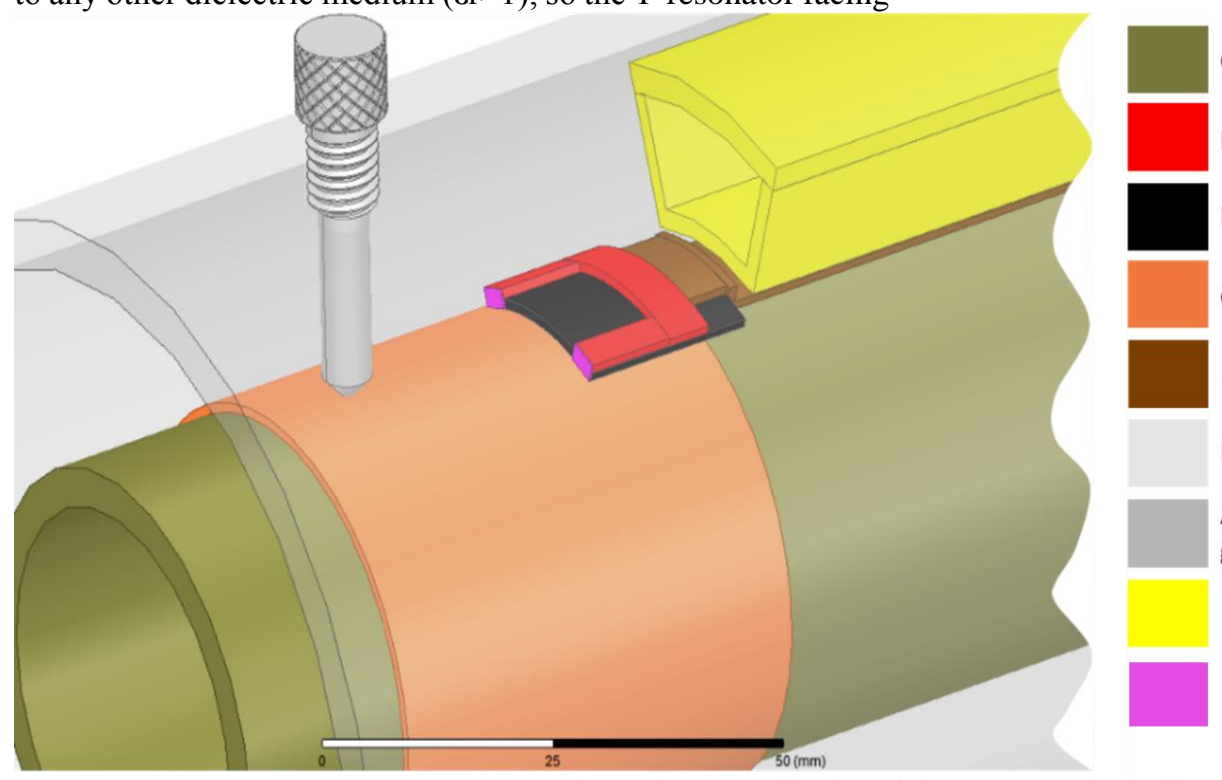

Central core made of acrylic

Feedline for microwave resonator

Dielectric separator

Ground plane for the feedline

$\lambda / 4$ shunt stub (electrically connected with feedline)

External metallic cylinder/Ground plane for $\lambda / 4$ Stub

Aluminium screw for shorting feedline $\& \lambda / 4$ stub ground planes

Liquid sensing area

Port for the feedline 
If we look at the $\mathrm{E}$ field distribution in the cross section of the proposed design containing a single resonator for now (shown in Fig. 4(a)), we can see that most of the fields are concentrated in $45^{\circ}$ wedge region between central core and external metallic cylinder. From this, we can deduce that the field of view of a single resonator is around $45^{\circ}$ and up to 8 similar resonators can be packed on the core to cover $360^{\circ}$ field of view (sensing). An interesting observation here is that the external metallic cylinder will act as a common ground plane for all the Tresonators, a key to a compact multi-level sensor.

However, there is a tendency for the E-fields of a single resonator to fringe to its neighboring region (shown in Fig. 4(a)). As a result, the resonance frequency of a single resonator, though mainly dependent upon the dielectric properties of the medium inside its $45^{\circ}$ region, is also slightly affected by the dielectric medium in the neighboring regions. In order to restrict the EM fields in $45^{\circ}$ sector, we have used the metallic "sector isolating sheets" separating $45^{\circ}$ sectors apart and dividing the cylindrical space into 8 sections as shown in Fig. 4(b).

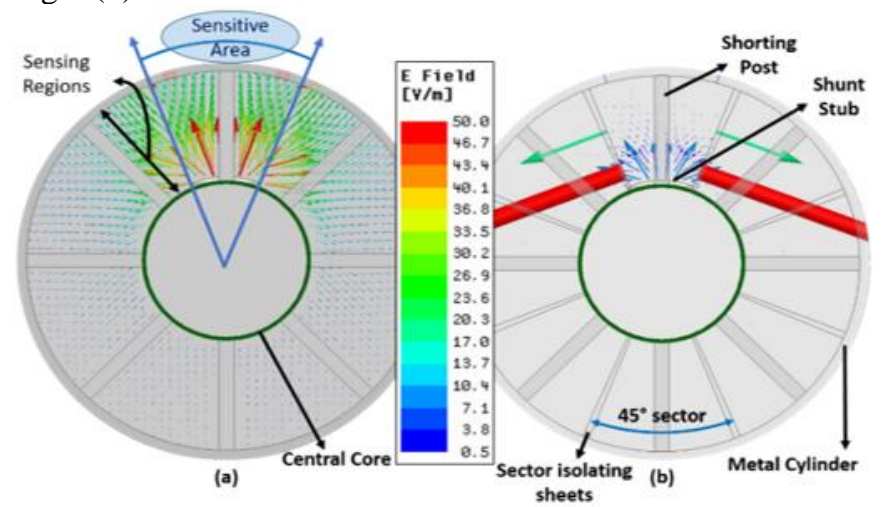

Fig. 4. Cross sectional view of the $\mathrm{E}$ field distribution in between central core and external metallic cylinder (a) Without the sector isolating sheets (b) with sector isolating sheets (The cross section shows 8 shorting posts i.e. one post for each resonator)

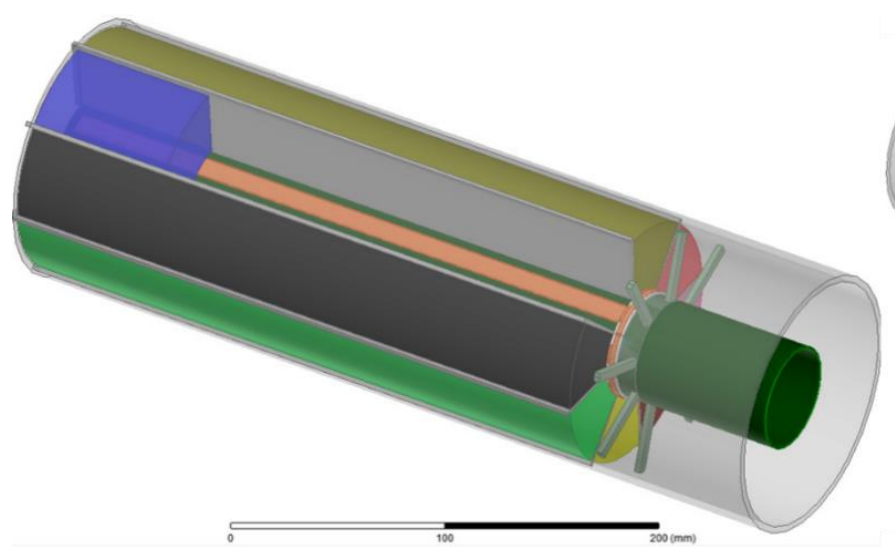

(a)
These sheets are shorted with the external cylinder making them part of the ground plane for the shunt stub. With the presence of 2 sheets on both sides of the resonator; E fields of a particular resonator will be restricted to its $45^{\circ}$ sector only (as evident from Fig. 4(b)) and the resonance frequency of each resonator will be dictated by the dielectric medium in that particular sector only which is a key to independent level measurements in neighboring liquid sectors.

In our case, these sectors are filled with different liquids. In an empty sector, air is filled inside a sector but as the liquid is poured inside a particular sector, effective dielectric constant (zeff) of the sector increases. It effectively decreases the resonance frequency as evident from eq. (1) and eq. (2). The change in resonance frequency can be correlated with the amount of liquid (level/volume) inside a sector.

In the proposed design; compact packing of 8 resonators inside the cylinder and its use as a common 3D ground plane enables simultaneous level sensing of 8 liquids. Moreover, the effective use of sector isolating sheets ensures that each liquid is sensed independent of its neighboring sectors. The final design of the proposed sensor is shown in Fig. 5 in which $2^{\prime \prime}$ internal core has been used inside a 4 " external metallic enclosure.

\section{FABRICATION}

The proposed design concept of a multi-level sensor has been validated by fabricating its prototype.

For the sensor prototype, central core was made of acrylic tube having an outer diameter of $50 \mathrm{~mm}$ and wall thickness of $2 \mathrm{~mm}$. Placeholder for printing ring ground plane for the feedlines was etched from the top surface of the central core as shown in Fig. 6(a). With the help of the 3D printed alignment mask, blue masking tapes were pasted on the acrylic tube to screen-print, eight (8) mutually aligned $\lambda / 4$ shunt stubs based resonators.

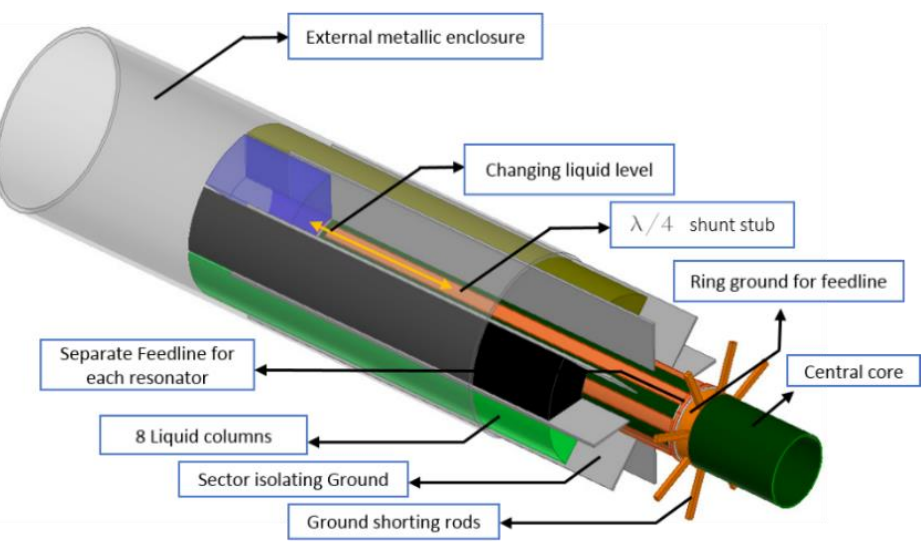

(b)

Fig. 5. (a) Proposed microwave sensor for simultaneous sensing of 8 liquid columns (b) Breakdown of the design 


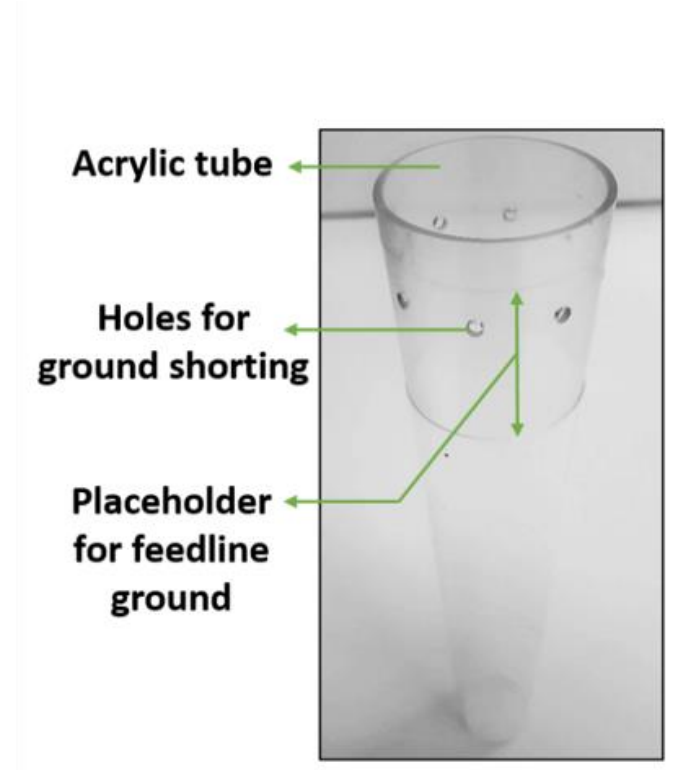

(a)

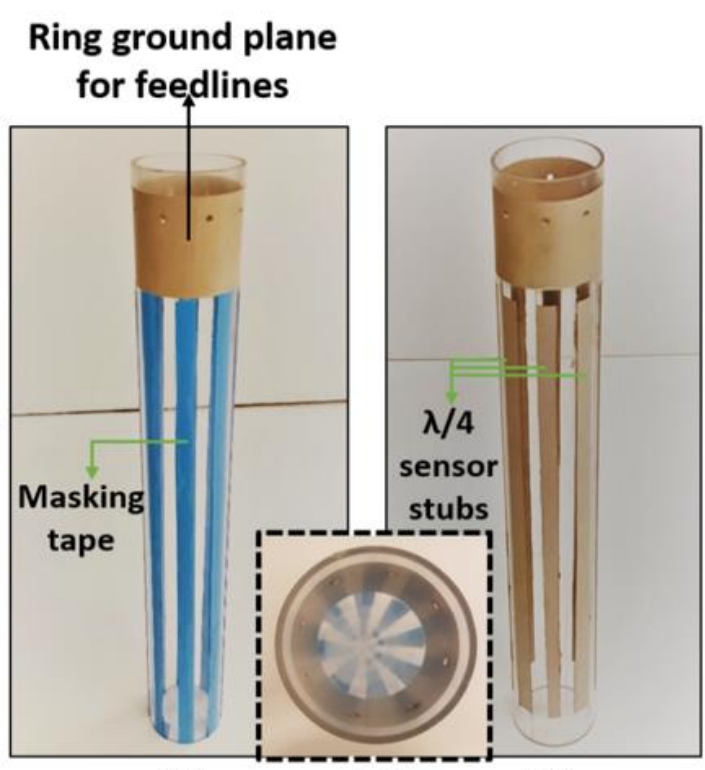

(b)

(c)

(d)

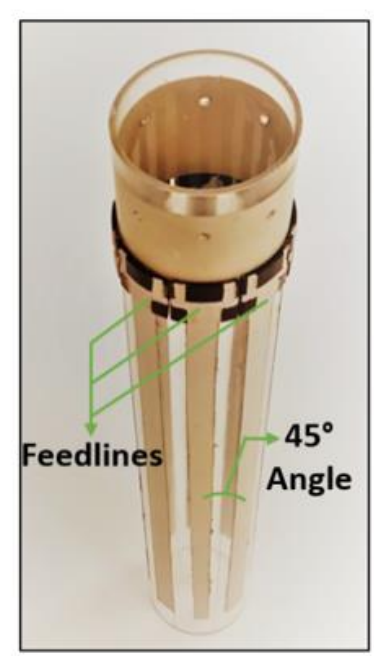

(e)

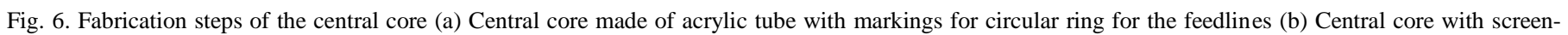

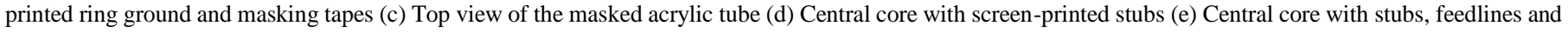
their 3D printed separators

Side view of the central core with masking tape is shown in Fig. 6(b) while its top view is shown in Fig. 6(c). Central core with eight (8) stubs screen-printed on it, looks like shown in Fig. 6(d). In order to feed microwave energy to these resonators, feedlines were printed on the 3D printed dielectric pieces (shown in black color in Fig. 6(e)) which also separates the feedline from its ring shaped ground plane lying underneath them.

Aluminum based outer metallic cylinder was machined (as shown in Fig. 7(a)) with eight slits in it. In order to isolate the $45^{\circ}$ sectors from each other, eight (8) aluminum based metallic strips were prepared, as shown in Fig. 7(b), which were diagonally inserted into the metallic cylinder and then conductively shorted to it. Eight dielectric containers were also 3D printed (as shown in Fig. 7(c)) to contain different liquids under test (LUT).

Eight ground shorting screws were also fabricated (as shown in Fig. 7(d)) to short the ring-shaped circular ground (acting as ground for the feedlines) with the external metallic cylinder (acting as common ground for the eight resonators).

Fully assembled prototype (with some non-visible parts) is shown in Fig. 7 with its side and top view shown in Fig. 7 (e), (f) respectively.

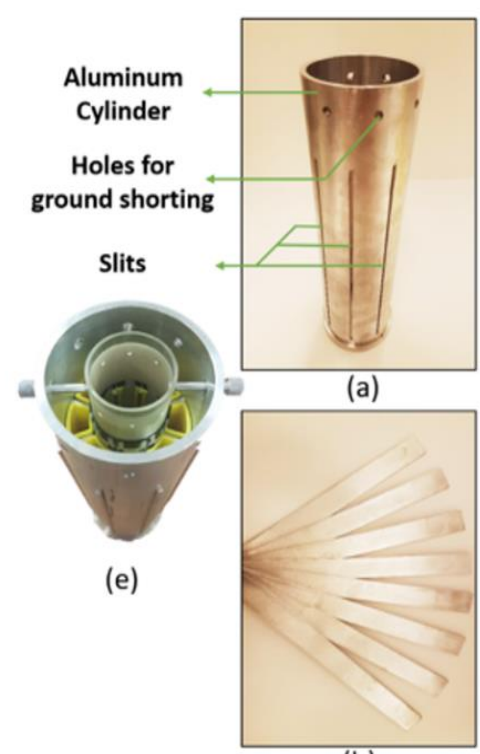

(b)

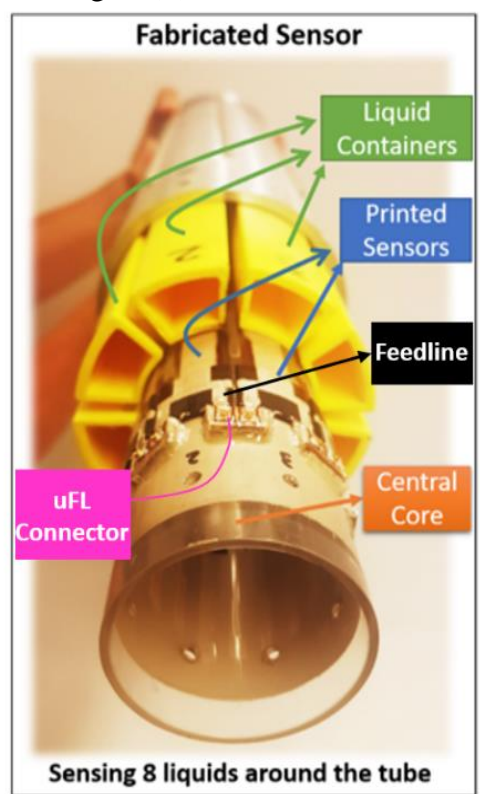

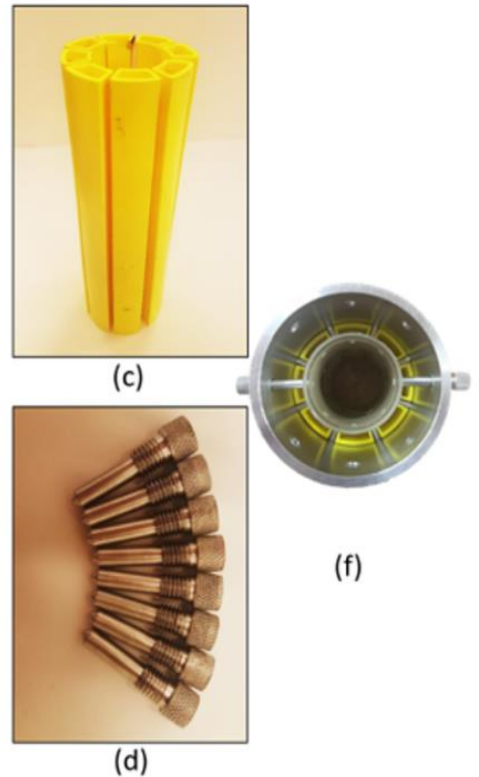

(d)

Fig. 7. Fully assembled prototype of the sensor (a) Aluminum based external cylinder (b) Aluminum based metallic strips for $45^{\circ}$ sector isolation (c) $3 \mathrm{D}$ printed container to contain liquids under test (LUT) (d) Aluminum based screws to short the circular ring ground (for feedlines) to the external metallic cylinder (ground for the resonators) (e) Side view of the assembled sensor (f) Top view of the assembled sensor 


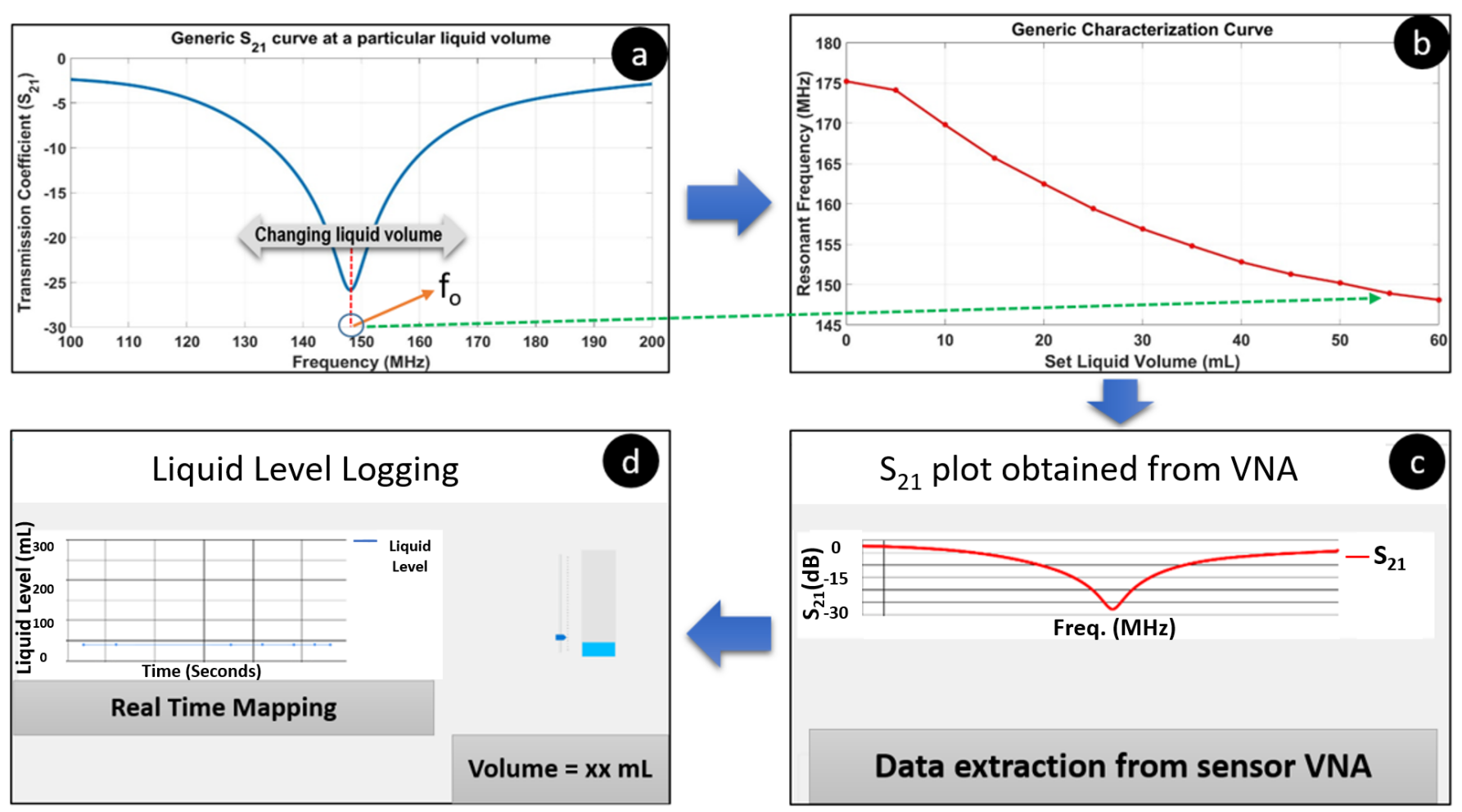

Fig. 8. Step by step flow of sensor operation (a) Step 1: S21 response of the sensor is recorded using VNA for different liquid volumes (b) Step 2: f0 extracted from the S21 response is interpolated against liquid volumes and this characterization curve is fed into the PC software (c) Step 3: Sensor is exposed to any unknown liquid amount and the sensor's S21 response is extracted by the software through Ethernet connection with the VNA (d) Step 4: PC software extracts the f0 from the S21 response and maps it with the interpolated characterization curve of the sensor for that particular liquid. The mapping converts f0 into the liquid volume in real time

\section{RESULTS}

Sensor operation and performance will be discussed in this section. Firstly, the sensor operation will be summarized in 4-step process which is used to obtain real time level measurements. Sensor is characterized in the first 2 steps of the process which will be discussed in detail in the following section. Characterization curves for the eight liquids form the database for real time measurements. In the last 2 steps of the process; sensor is exposed to unknown liquid levels and a computer software reads the real time level of the liquid by matching the sensor's response to the database already fed into the software. In the end, sensor calibration procedure will be detailed and its effect on sensor's performance will be presented.

\section{A. Operational flow of the sensor}

As highlighted in the section of "sensing principle" that the resonant frequency of the modified T-resonator changes with the liquid level or volume which is measured using 2-port (between P1 and P2 of Fig. 1) transmission parameter (S21) of the T-resonator using N9923A handheld vector network analyzer (VNA) by Keysight Technologies.

Sensing the level of a particular liquid requires four steps which have been summarized in Fig. 8. First of all, a particular liquid compartment is filled up with different amounts of liquid and the transmission coefficient (S21) curve over the frequency range of $100 \mathrm{MHz}$ to $200 \mathrm{MHz}$ is recorded using VNA. It should be noted here that the T-resonator has a band-stop response which means that the microwave energy is blocked from one port to the other at the resonant frequency $\left(\mathrm{f}_{0}\right)$ and the minimum valley point is denoted as the $\mathrm{f} 0$ of the sensor for a particular liquid level. S21 curve for a particular liquid level is shown in Fig. 8(a).

Since, eeff is increased with increasing amount of liquid inside a liquid container, causing fo to decrease. This change in $\mathrm{f0}$ is plotted against liquid volume (as shown in Fig. 8(b)) and this characterization table is fed into the PC software. These two characterization steps need to be done only once for each liquid.

After that, liquid compartment is exposed to any unknown liquid level. The S21 response obtained from VNA is transferred to the PC software through Ethernet cable as shown in Fig. 8 (c). The resonant frequency is extracted from the S21 curve and is mapped to the characterization curve of that particular liquid which is already fed into the software. This way software converts the fo into "measured liquid volume/level" in real time as shown in Fig. 8 (d).

\section{B. Sensor characterization}

The first step to get this sensor to work is to characterize it for the intended list of liquids. Eight liquid compartments are filled up with a volume from $0 \mathrm{~mL}$ to $60 \mathrm{~mL}$ with a step size of $5 \mathrm{~mL}$. 


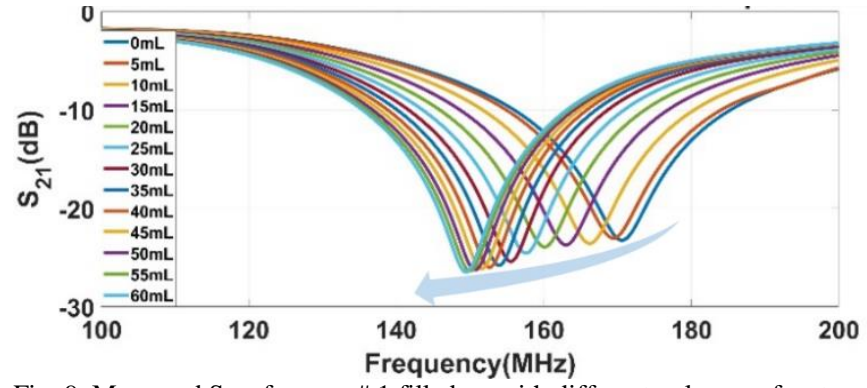

Fig. 9. Measured $S_{21}$ of sensor \# 1 filled up with different volumes of water

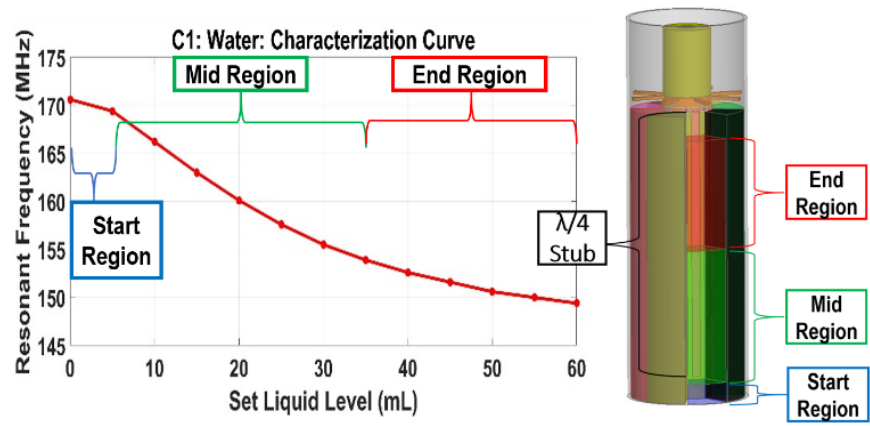

Fig. 10. Characterization curve obtained from sensor \#1 with water liquid

Fig. 9 shows the S21 response curves of sensor (resonator) \#1 for different liquid volumes of water used in container \#1. It can be confirmed from this figure that the resonance frequency decreases with increasing amount of water volume. The resonant frequency was extracted from these curves and plotted against the liquid volume to form the characterization curve for water in container \#1 as shown in Fig. 10 which acts as a lookup table for real time measurement of any unknown liquid amount.

The characterization curve, shown in Fig. 10, can be divided into 3 regions i.e. start region, mid region and end-region. Start region ranges from $0 \mathrm{~mL}$ to $5 \mathrm{~mL}$ in volume (or $0 \mathrm{~mm}$ to $24 \mathrm{~mm}$ in level) where the sensitivity of the sensor is relatively low because this is where only the weak fringing fields of the shunt stub exists. This region can be further minimized by either extending the length of the stub a bit or increasing the bottom base thickness of the liquid container. Then comes the mid region ranging from $5 \mathrm{~mL}$ to $35 \mathrm{~mL}$ (or $25 \mathrm{~mm}$ to $170 \mathrm{~mm}$ ) where the sensor shows the linear change in resonant frequency w.r.t. liquid level. In the end comes the "end region" which ranges from $35 \mathrm{~mL}$ to $60 \mathrm{~mL}$ (or $170 \mathrm{~mm}$ to $290 \mathrm{~mm}$ ) where the sensitivity of the sensor is lesser compared to the mid region. Although both the mid and end regions lie facing the shunt stub but the sensitivity of the "end region" is lesser compared to the mid region because the percentage change in effective dielectric constant (ceff) gradually decreases as the container is filled up with the liquid.

Similar characterization curves for 7 other resonators with 7 other liquids i.e. Methanol, 1-Butanol, 2N Butoxy Ethanol, 2Butanol, Ethanol, IPA and vegetable oil have also been obtained. The S21 responses and the characterization curves of all the liquids have been included as Fig. 16 and Fig. 17 in the Appendix section. From those characterization curves, it can be observed that all the curves have similar shape which can be divided into same 3 regions as mentioned above. It can also be observed from those curves that the liquids having higher dielectric constant like Methanol show more percentage change in resonant frequency compared to the liquids with lower dielectric constant like vegetable oil. However, the starting point (air response) of these curves are not exactly the same, mainly because of the fabrication imperfections which can be minimized with precise industrial machining processes.

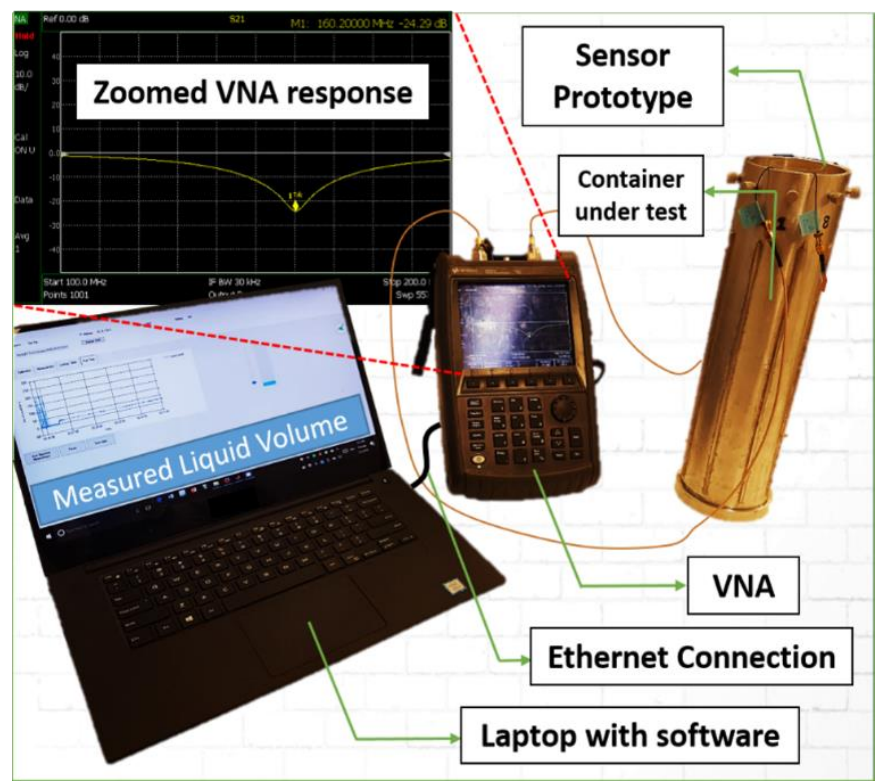

Fig. 11. Test setup to measure the unknown liquid amount

\section{Real Time Sensor Operation}

The characterization curves of all the eight sensors were fed into the PC software which were used as the lookup tables for measuring unknown liquid amount. Appropriate characterization curve can be selected in the software based upon the LUT. The test setup for measuring the unknown liquid amount is shown in Fig. 11. As the liquid is poured into the container, the connected VNA records the S21 curve which is read by the software using Ethernet connection. Software extracts the resonant frequency from the S21 curve and maps it with the appropriate lookup table and transforms the reading into the measured liquid volume. Real time reading of the measured liquid level is then displayed on the time scale which can be used for process automation or can also be exported and used for data analytics.

Sensor performance has been tested for all the eight liquids by following the steps mentioned earlier. Although the testing on the eight sensors was performed sequentially, however, two "Single Pole Eight Throw (SP8T)" MMIC based multiplexers [22] can easily be integrated with the sixteen ports of the sensor to simultaneously sense eight liquid levels.

While evaluating the performance of the sensor; it was exposed to any unknown liquid volume in the range of $0 \mathrm{~mL}$ to $60 \mathrm{~mL}$ which is plotted on $\mathrm{x}$-axis of Fig. 12. While the sensor measures the liquid level using the procedure mentioned above and is plotted on the y-axis of Fig. 12.

The green line represents the ideal case if the measured liquid level is exactly equal to the set liquid level. It can be seen 


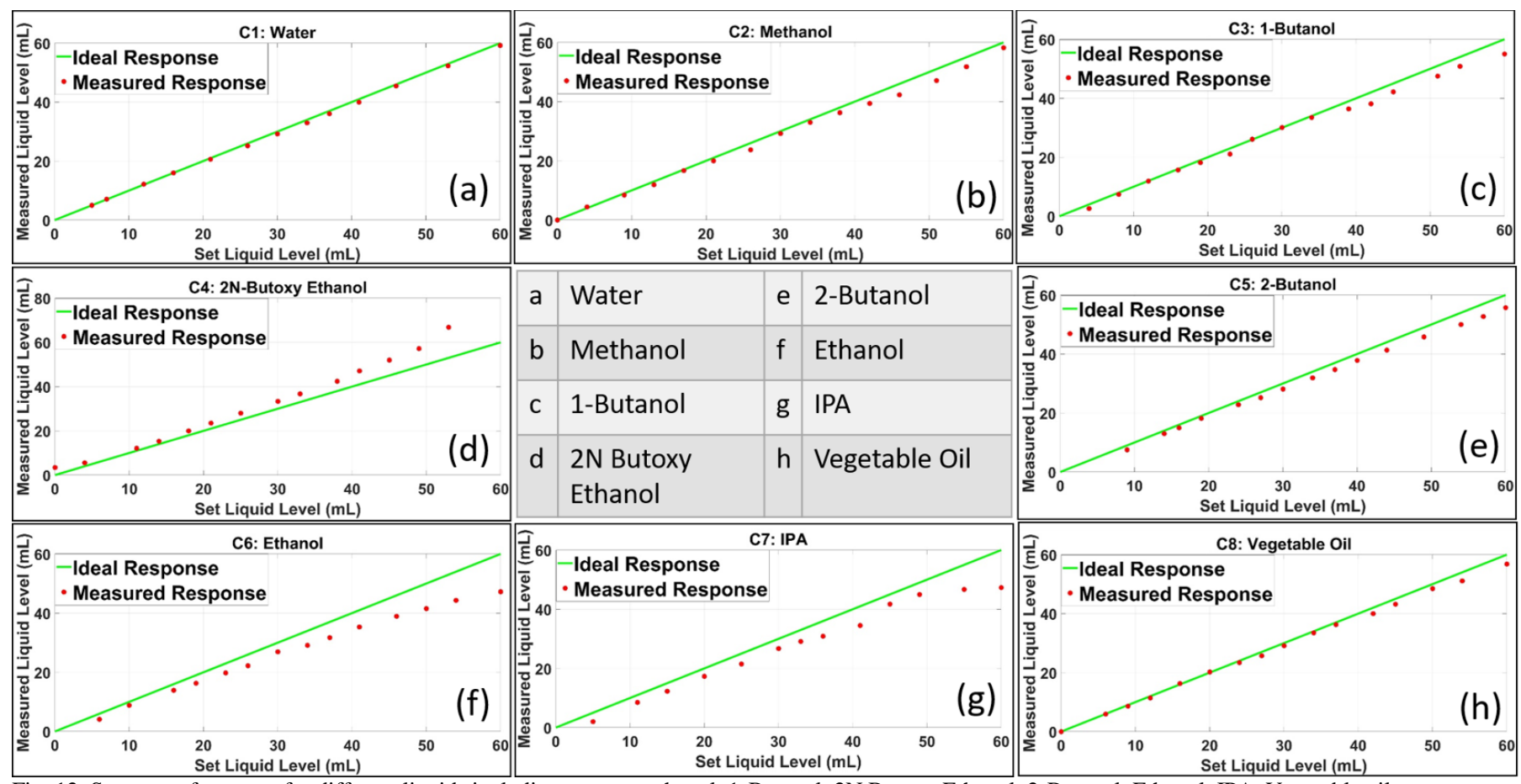

Fig. 12. Sensor performance for different liquids including water, methanol, 1-Butanol, 2N Butoxy Ethanol, 2-Butanol, Ethanol, IPA, Vegetable oil

from Fig. 12 that most of the red dots, which are the actual measured data points, are very close to the ideal green curve. Any deflection of red dot from the green curve represents the error in measurement. It can be seen from all the measurement curves that the measurement error is very minor for sensing low liquid levels while it exponentially grows as the liquid volume to be sensed is increased for some liquids.

It is worth mentioning here that the liquid compartments are not tightly packed so that they can be easily removed for servicing. However, it also results into their misalignment w.r.t. the resonator, every time they are put back into the sensing unit after servicing. This misalignment causes a fixed shift (offset) in sensor's response to either low or higher frequency which means that the whole characterization curve shifts down or up respectively.

The constant offset is not so detrimental while measuring low amounts of liquid (in the mid region) but the error exponentially grows when it comes to measuring larger liquid volumes (in the end region). This is because the constant offset results into less

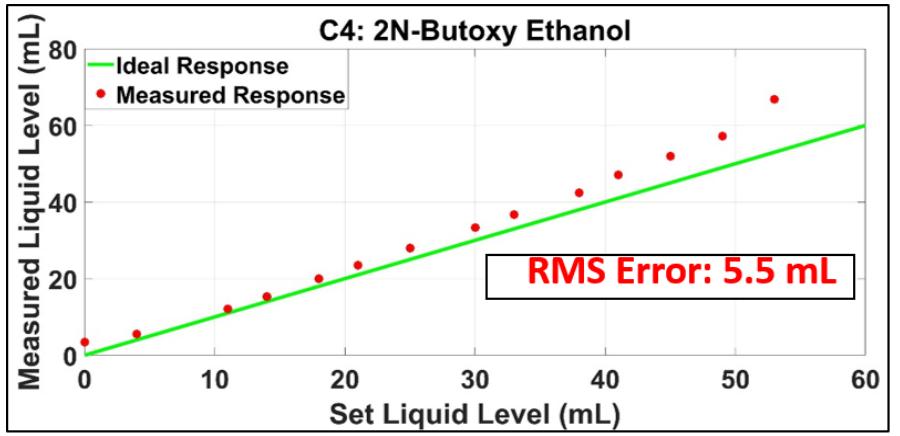

(a) percentage error in the mid region because of higher sensitivity there while the same offset results into high percentage error due to low sensitivity of the sensor there. This kind of error can be compensated by the constant offset calibration which is explained in next section.

\section{ONE POINT SENSOR CALIBRATION}

As explained earlier that the kind of error observed for some of the liquids is because of constant offset. This offset can be measured by differencing the resonant frequencies obtained with the empty container for the case when the sensor is initially characterized (say it is $\mathrm{f}_{0 \mathrm{c}}$ ) and whenever real time measurements are performed on unknown liquid amounts (say it is $\left.\mathrm{f}_{0 \mathrm{u}}\right)$. The offset measured using eq. 3 is then subtracted from the characterization curve resulting into calibrated curve or lookup table.

$$
\text { Offset }=\mathrm{f}_{0 \mathrm{c}}-\mathrm{f}_{0 \mathrm{u}}
$$

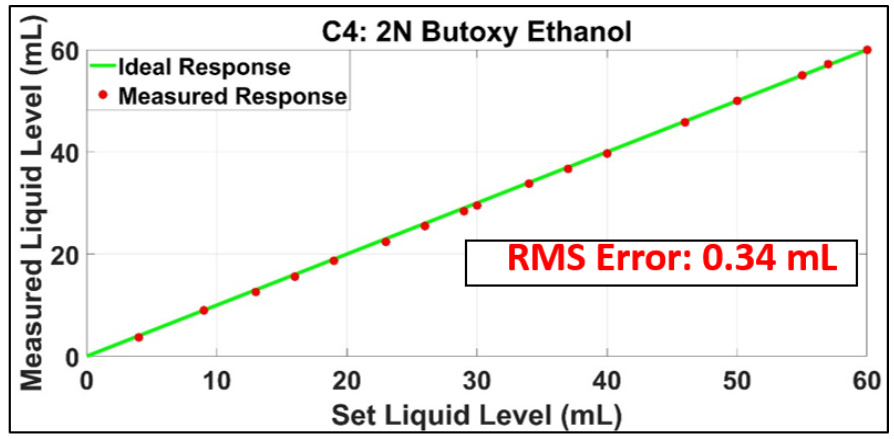

(b)

Fig. 13. Effect of one point calibration on the sensor's performance (a) sensor performance without calibration (b) sensor performance with calibration 
To see the effectiveness of the proposed one point calibration, calibrated lookup table was tested with container \# 4 containing $2 \mathrm{~N}$ Butoxy Ethanol. Fig. 13 shows the comparison of the sensor performance to measure unknown volume of $2 \mathrm{~N}$ Butoxy Ethanol liquid with and without calibration. It can be seen from the figure that the RMS error in measuring the liquid volume has been decreased from $5.5 \mathrm{~mL}$ to $0.34 \mathrm{~mL}$ ( $0.6 \%$ error) which is an improvement of more than $16 x$ due to the calibration. The proposed one point calibration is also very simple to apply in industrial settings in which sensor's response is to be measured once in the empty container before starting the level measurement.

\section{CONCLUSION}

The article has presented the design and implementation of a new type of multi-channel level sensor which in addition to providing accurate level measurements, can also pack multiple level sensors in a very compact form factor. For the first time ever, microwave T-resonance phenomenon has been demonstrated to operate effectively inside a metallic cylinder utilizing it as a common 3D ground plane for eight resonators. In future, MMIC based multiplexers can be integrated with the resonators to automate the process of simultaneous level measurements of multiple liquids. RF oscillator can further be integrated with the resonators to eliminate the need of VNA in order to develop a self-contained sensor for multiple and simultaneous level measurements.

\section{REFERENCES}

[1] P. Raatikainena, I. Kassamakovb, R. Kakanakovb and M. Luukkalaa, "Fiber-optic liquid-level sensor," Sensors and Actuators A: Physical, vol. 58, no. 2, pp. 93-97, 1997.

[2] P. Hauptmann, N. Hoppe and A. Püttmer, "Application of ultrasonic sensors in the process industry," Measurement Science and Technology, vol. 13, no. 8, 2002.

[3] J. Terzica, C.R.Nagarajahb and M. Alamgir, "Fluid level measurement in dynamic environments using a single ultrasonic sensor and Support Vector Machine (SVM)," Sensors and Actuators A: Physical, vol. 161, no. 1-2, pp. 278-287, 2010.

[4] G. R. N. M. Brajesh Kumar, "A review on Capacitance-Type Sensor for Measurement of Height of Liquid Level," Measurement and Control, vol. 47, no. 7, pp. 219-224, 2014.

[5] X. L. Q. B. D. W. Y. W. Baoquan Jin, "Design and Implementation of an Intrinsically Safe Liquid-Level Sensor Using Coaxial Cable," Sensors, vol. 15, no. 6, pp. 12613-12634, 2015.

[6] "Design and Demonstration of a RADAR Gauge for In-Situ Level Measurement in Furnace," IEEE Sensors Journal, no. 99, 2018.

[7] E. Denicke, G. Armbrecht and I. Rolfes, "A correlation-based method for precise radar distance measurements in dispersive waveguides," in European Radar Conference, EuRAD, Rome, 2009.

[8] J. Otto, "Radar Applications in Level Measurement, Distance Measurement and Nondestructive Material Testing," in European Microwave Conference, Jerusalem, 1997.

[9] M. Demori, V. Ferrari, D. Strazza and P. Poesio, "A capacitive sensor system for the analysis of two-phase flows of oil and conductive water," Sensors and Actuators A: Physical, vol. 163, no. 1, pp. 172-179, 2010.

[10] T. Chretiennot, D. Dubuc and K. Grenier, "A Microwave and Microfluidic Planar Resonator for Efficient and Accurate Complex Permittivity Characterization of Aqueous Solutions," IEEE Transactions on Microwave Theory and Techniques, vol. 61, no. 2, pp. 972 - 978, 2012.
[11] A. A. Abduljabar, N. Clark, J. Lees and A. Porch, "Dual Mode Microwave Microfluidic Sensor for Temperature Variant Liquid Characterization," IEEE Transactions on Microwave Theory and Techniques, vol. 65, no. 7, pp. 2572 - 2582, 2017

[12] FlowLine, "Liquid \& Solids Level Transmitters," [Online]. Available: https://www.flowline.com/level-transmitters/. [Accessed 21 April 2018].

[13] YouTube, National Bulk Equipment, 29 May 2014. [Online]. Available: https://www.youtube.com/watch?v=dFes-spxKx4.

[14] YouTube, Schenck Process UK, 16 August 2012. [Online]. Available: https://www.youtube.com/watch?v=ezk2h8AAIoY.

[15] M. A. Karimi, M. Arsalan and A. Shamim, "A low cost, printed microwave based level sensor with integrated oscillator readout circuitry," in IEEE MTT-S International Microwave Symposium (IMS), 2017, Honololu, HI, USA, 2017.

[16] M. A. Karimi, M. Arsalan and A. Shamim, "Live demonstration: Screen printed, microwave based level sensor for automated drug delivery," in 2017 IEEE SENSORS, Glasgow, 2017.

[17] M. A. Karimi, M. Arsalan and A. Shamim, "Design and Dynamic Characterization of an Orientation Insensitive Microwave Water-Cut Sensor," IEEE Transactions on Microwave Theory and Techniques, vol. 66, no. 1, pp. 530-539, 2017.

[18] M. A. Karimi, M. Arsalan and A. Shamim, "Low Cost and Pipe Conformable Microwave-Based Water-Cut Sensor," IEEE SENSORS JOURNAL, pp. 7636-7645, 2016

[19] M. A. Karimi, M. Arsalan and A. Shamim, "A low-cost, orientationinsensitive microwave water-cut sensor printed on a pipe surface," in IEEE MTT-S International Microwave Symposium (IMS), Honololu, 2017

[20] Microwaves101, "Light, Phase and Group Velocities," [Online]. Available: https://www.microwaves101.com/encyclopedias/lightphase-and-group-velocities. [Accessed 21 April 2018].

[21] M. K. J.-P. S. P. S. K.-P. Lätti, "A Review of Microstrip T-Resonator Method in Determining the Dielectric Properties of Printed Circuit Board Materials," IEEE TRANSACTIONS ON INSTRUMENTATION AND MEASUREMENT, vol. 56, no. 5, pp. 1845-1850, 2007.

[22] H. M. Cooperation, "HMC253ALC4," [Online]. Available: http://www.analog.com/en/products/rf-microwave/rf-switches/spstspdt-sp3t-sp4t-sp6t-sp8t/hmc253alc4.html\#product-overview. [Accessed 23 April 2018]. 


\section{APPENDIX}

A. Dielectric measurements of liquids under test

The sensor has been tested with eight different types of liquids commonly available in the lab. Although those liquids were used for experimentation which did not have significant chemical interaction with the $3 \mathrm{D}$ printed liquid containers, however, there is no restriction on the type of liquid which can be tested with this sensor as long as the liquid container is made of appropriate dielectric material compatible with its container.

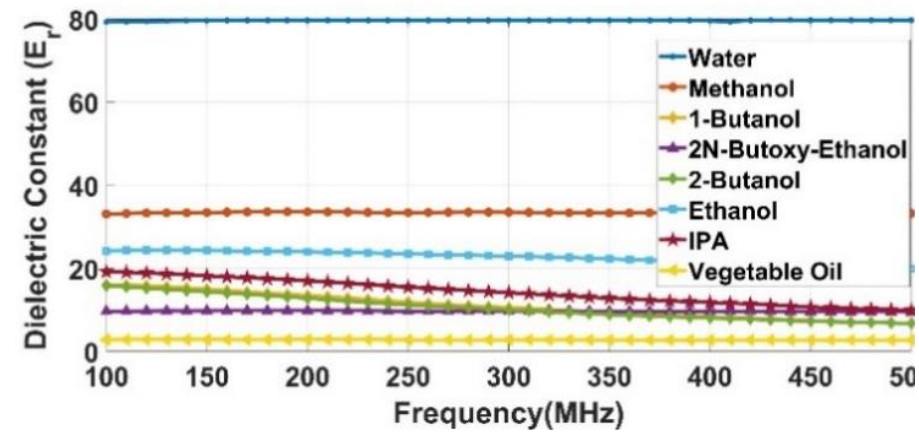

Fig. 14. Measured dielectric constant of 8 liquids tested with the proposed level sensor

Eight liquids tested for the experimentation are Water, Methanol, 1-Butanol, 2N Butoxy Methanol, 2-Butanol,
Ethanol, IPA and vegetable oil. The dielectric properties (dielectric constant and dielectric loss tangent) of these liquids have been first measured using dielectric assessment kit (DAK12) from $100 \mathrm{MHz}$ to $500 \mathrm{MHz}$ (shown in Fig. 14 and Fig. 15) while the operational frequency of the resonators are in the frequency band of $130 \mathrm{MHz}-180 \mathrm{MHz}$ depending upon the dielectric constant of the liquid under test.

It can be seen from Fig. 14 that the LUT have dielectric constant ranging from 2.7 to 80 while Fig. 15 shows that the dielectric loss of almost all the mediums increases with frequencies.

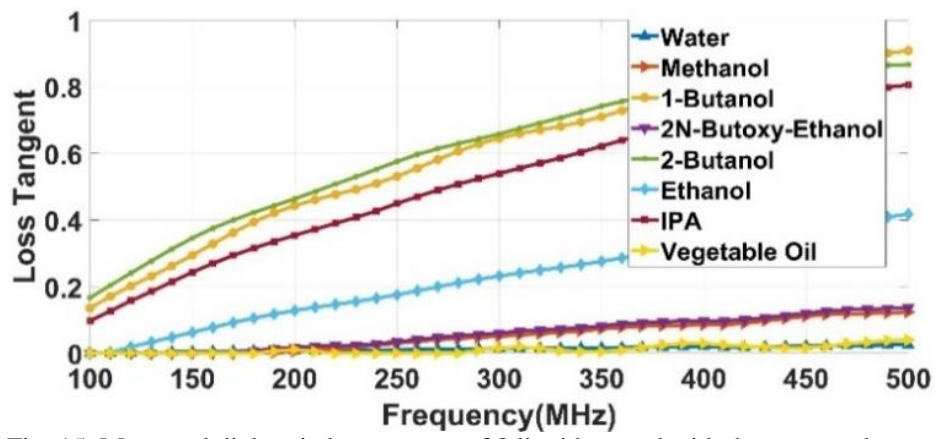

Fig. 15. Measured dielectric loss tangent of 8 liquids tested with the proposed level sensor

\section{B. S21 response of the sensor for 8 different liquids}

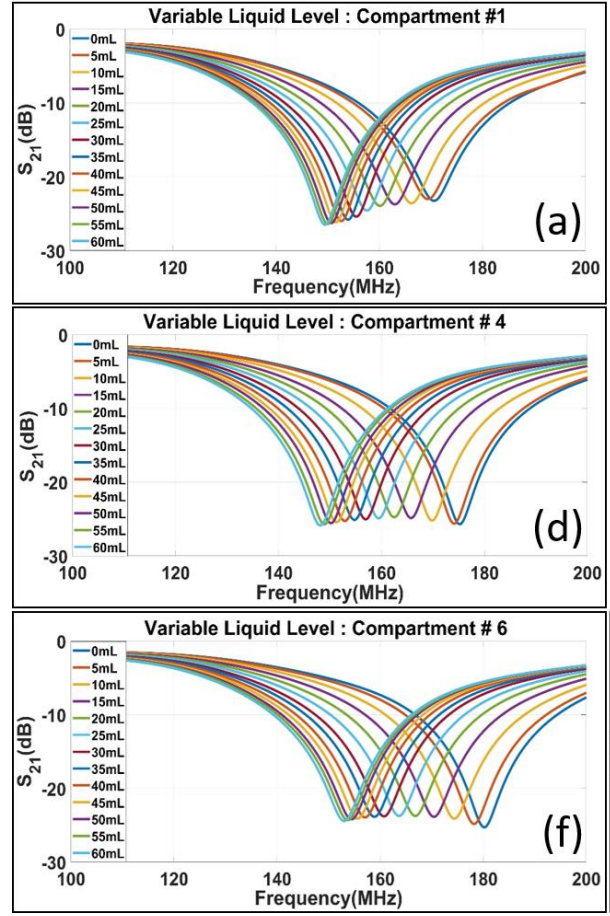

(a)

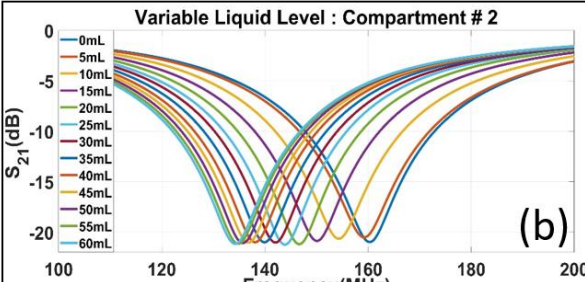

(b)

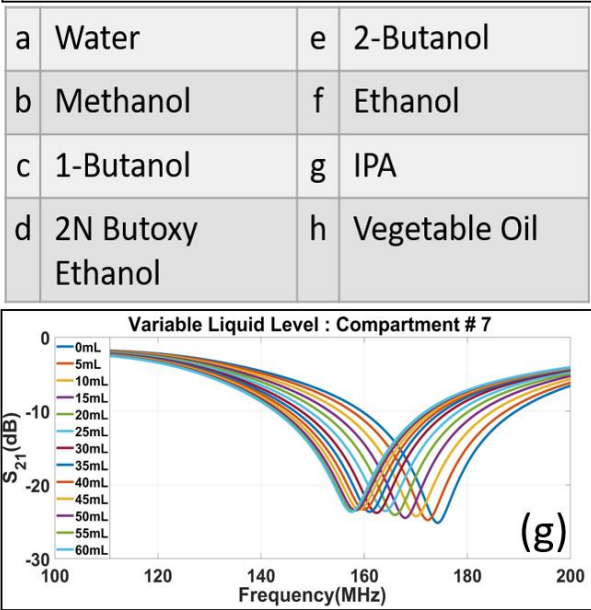

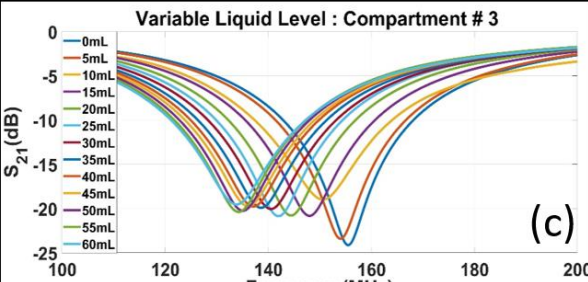

(c)

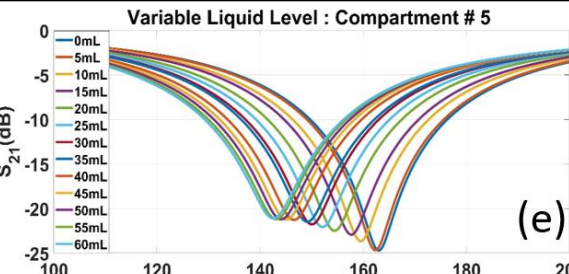

(e)

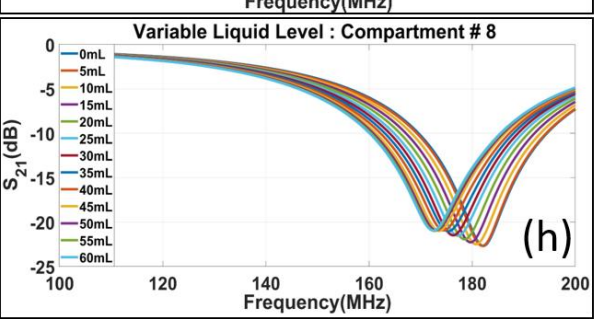

Fig. 16. S21 responses of 8 sensor channels to 8 liquids for different liquid levels ranging from $0 \mathrm{~mL}$ to $60 \mathrm{~mL}$ 


\section{Characterization curves formed for 8 different liquids}

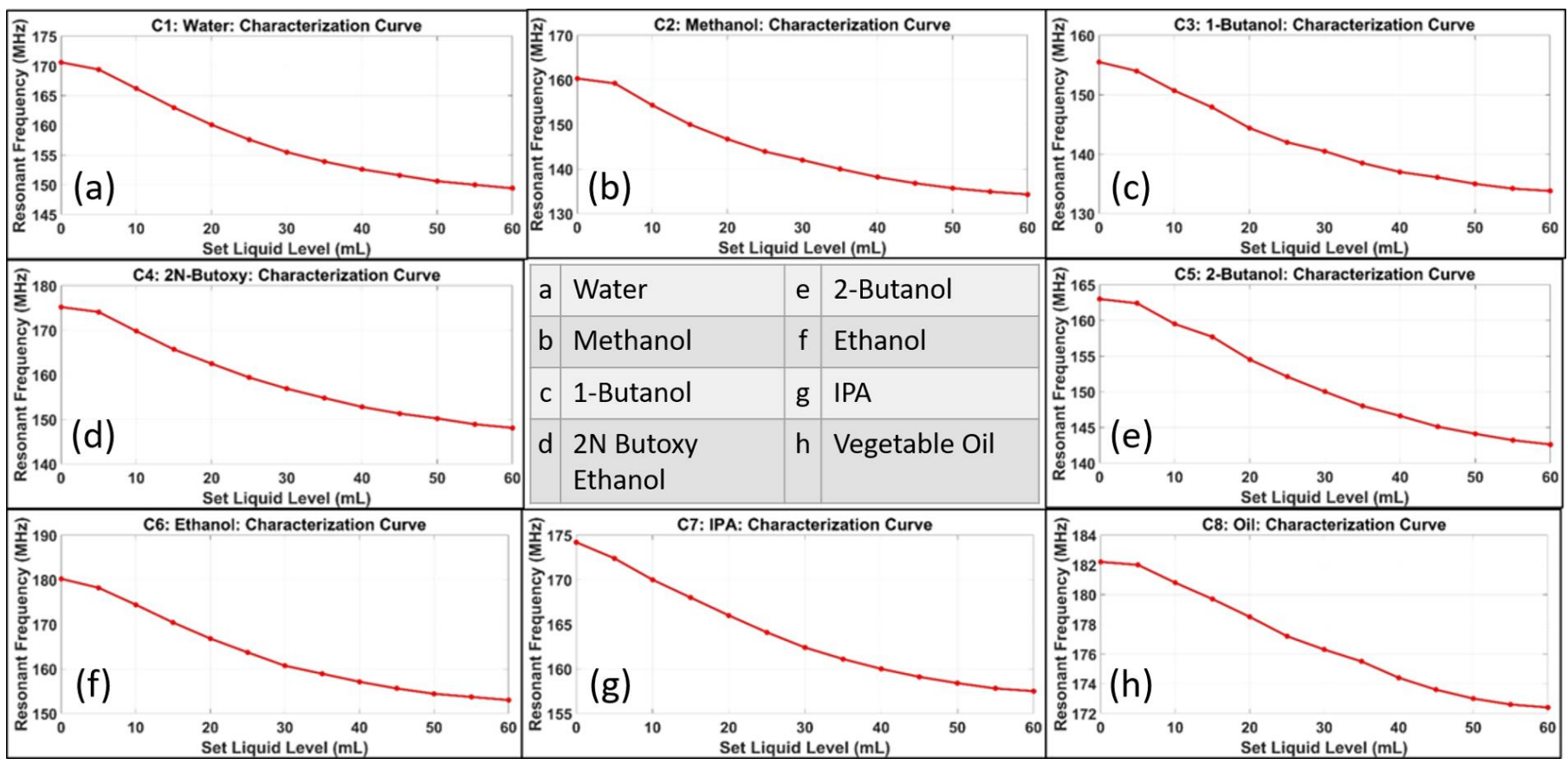

Fig. 17. Characterization curves obtained from 8 sensor channels when tested with 8 liquids 